\title{
Stevens-Johnson Syndrome
}

National Cancer Institute

\section{Source}

National Cancer Institute. Stevens-Johnson Syndrome. NCI Thesaurus. Code C79484.

A systemic, serious, and life-threatening disorder characterized by lesions in the skin and mucous membranes that may lead to necrosis. The lesions may appear anywhere in the body but they occur more commonly in the palms, soles, dorsum of the hands, and extensor surfaces. The lesions are vesicular or necrotic in the center, surrounded by an erythematous zone and occupy less than $10 \%$ of the body surfaces. The appearance of the mucocutaneous lesions is preceded by an upper respiratory tract infection. It is an immune complex hypersensitivity reaction usually caused by drugs (e.g., sulfa, phenytoin, penicillin), viruses (e.g., herpes simplex, influenza, hepatitis), and malignancies (e.g., carcinoma and lymphoma). 